\title{
Calidad de vida en pacientes costarricenses con tumores cerebrales: aportes de la neuropsicología
}

\author{
Quality of Life in Costa Rican Patients with Brain Tumors: \\ Neuropsychological Contributions
}

\author{
Mónica Salazar Villanea ${ }^{1}$ \\ Luis Enrique Ortega Araya ${ }^{2}$ \\ Jorge Ortiz Álvarez ${ }^{3}$ \\ Universidad de Costa Rica, Costa Rica
}

\author{
Miguel Angel Esquivel Miranda ${ }^{4}$ \\ Rocío Vindas Montoya ${ }^{5}$ \\ Patricia Montero Vega ${ }^{6}$ \\ Hospital México, Costa Rica
}

Resumen. Se describe una experiencia clínico-formativa en el ámbito hospitalario, donde destacan los aportes de la neuropsicología en la atención multidisciplinaria a pacientes con tumores cerebrales. Se ejemplifican cuatro casos atendidos en el Servicio de Neurocirugía del Hospital México en Costa Rica, a quienes se les realizó una valoración neuropsicológica como parte del protocolo de atención en cirugía de resección tumoral. Se documenta una leve pero consistente tendencia a la mejoría en los rendimientos tras la intervención neuroquirúrgica de los casos reportados, evidenciando, también, las secuelas neurocognitivas y emocionales que permanecen e impactan directamente la calidad de vida. Se discute sobre los retos asociados a la expectativa sanitaria de prolongación de la vida pero preservando su calidad y funcionalidad.

Palabras clave. Neuropsicología, tumor cerebral, neurocirugía, calidad de vida, evaluación, déficits neuropsicológicos, rehabilitación cognitiva.

Abstract. The systematization of a clinical and educational experience in a hospital setting shows evidence of the neuropsychological contributions to the multidisciplinary care for patients with brain tumors. Four cases of people diagnosed with brain tumors are exemplified. The patients were treated at the Neurosurgical Unit at the Hospital Mexico, Costa Rica. Each patient underwent neuropsychological evaluations as part of the established protocol for neurosurgical interventions. Results showed a slight yet consistent trend of cognitive performance improvement after each surgery, and identified residual neurocognitive consequences that would have a direct impact on the patients quality of life. This paper discusses the challenges associated with health expectancy of prolonging life while preserving its quality and functionality.

Keywords. Neuropsychology, brain tumor, neurosurgery, quality of life, assessment, neuropsychological deficitscognitive rehabilitation.

\footnotetext{
${ }^{1}$ Mónica Salazar Villanea. Universidad de Costa Rica, Costa Rica. Dirección postal: IIP-UCR: 11501-2060 E-mail: monica.salazarvillanea@ucr.ac.cr

${ }^{2}$ Luis Enrique Ortega Araya Universidad de Costa Rica, Costa Rica. E-mail: luisenriqueoa@gmail.com

${ }^{3}$ Jorge Ortiz Álvarez. Universidad de Costa Rica, Costa Rica. E-mail: jorgeisaaco@hotmail.com

${ }^{4}$ Miguel Angel Esquivel Miranda. Hospital México, Costa Rica. E-mail: miguelesquivel09@gmail.com

${ }^{5}$ Rocío Vindas Montoya. Hospital México, Costa Rica. E-mail: rociovindas@yahoo.com

${ }^{6}$ Patricia Montero Vega. Hospital México, Costa Rica. E-mail: pmonterohm@ccss.sa.cr
}

\section{(@) $\odot \Theta \Theta$}

Esta obra está bajo una licencia de Creative Commons Reconocimiento-NoComercial-SinObraDerivada 4.0 Internacional. 


\section{Introducción}

Amplias discusiones científicas, académicas, sociales y éticas giran en torno al dilema de la posibilidad de prolongación de la vida versus la garantía real de una calidad de vida asociada, como sucede en la toma de decisiones neuroquirúrgicas en los ámbitos hospitalarios (Schagen et al., 2014). En este sentido, el rol de la psicología y la neuropsicología se posicionan como fundamentales para apoyar una recuperación integral, con opciones para identificar y compensar posibles secuelas (Dwan, Ownsworth, Chambers, Walker \& Shum, 2015), así como para afrontar los cambios relacionados con el proyecto de vida.

Las decisiones sobre los procesos integrales de atención en el ámbito de la salud deben basarse en la evidencia científica internacional (Spring, 2007) y la articulación de la experiencia y competencia clínica multidisciplinaria, así como la atención a las necesidades, valores y expectativas expresas de los y las pacientes (Shedden-Mora, 2014).

En Costa Rica, estos tres componentes anteriormente mencionados sirven como pilares de una experiencia clínico-formativa que, desde el año 2012, se desarrolla de forma continua entre la Escuela de Psicología de la Universidad de Costa Rica y los Servicios de Neurocirugía y Neurooncología, así como Psiquiatría y Psicología del Hospital México. Mediante la modalidad de Práctica Dirigida como Trabajo Final de Graduación, se ha realizado la inserción supervisada de estudiantes avanzados de último año de Licenciatura de la carrera de Psicología para asistir la demanda de atención de pacientes neuroquirúrgicos y sus familias (Ortega, 2013; Ortiz, 2014; Quesada, 2015 ; Molinari, 2015). Se han apoyado funciones como: a.) valoración psicológica y neuropsicológica antes, durante y después de las cirugías de resección tumoral, b.) acompañamiento y preparación de pacientes para los procesos neuroquirúrgicos, c.) valoración de posibles secuelas neuropsicológicas, d.) intervención clínica y e.) psicoeducación y acompañamiento tanto para el paciente como para su familia.
Las neoplasias (masas tumorales de tejido) constituyen una de las principales causas de muerte en el mundo (OMS, 2012) y la psicología ha cumplido un rol de relevancia en el estudio y atención de las personas y familias que han afrontado estos procesos de enfermedad (Brannon, 2007; Alvarán, Gómez, Aguirre \& Ortiz, 2008). Específicamente, en el caso de los tumores cerebrales o neoplasias del Sistema Nervioso Central (SNC), existe una amplia documentación sobre la diversidad de alteraciones cognitivas, comportamentales, emocionales y físicas en las personas que lo padecen. Es competencia de la neuropsicología clínica la intervención oportuna, por sus aportes a la comprensión y manejo de las implicaciones psicosociales de los déficits y sus herramientas para brindar rehabilitación a personas que presentan deterioro cognitivo. Para ello, considera la complejidad de los impactos en la experiencia personal, familiar, social y económica, donde los cursos tan diversos de la evolución del deterioro no se explican solamente por el daño neurológico sino por la influencia de factores de vida ambientales y personales (Salazar-Villanea, 2012).

A nivel mundial en el 2012 se registró una incidencia de 256213 nuevos casos de tumores cerebrales, según los datos de Globocan de la Organización Mundial de la Salud (OMS, 2012), con un total de 189394 defunciones. En Estados Unidos, el Instituto Nacional del Cáncer (CNI, por sus siglas en inglés), indicó 23380 nuevos casos para el 2014 en ese país. Según Alvarán et al. (2008), los tumores cerebrales son la segunda causa de muerte en las personas menores de 35 años, presentándose una mayor incidencia en hombres que en mujeres. En Costa Rica, Esquivel, Vargas, Alonso, Fernández, Jiménez y Cabezas (2012) señalan que del total de muertes por neoplasias en el país, un 2.67\% correspondieron a neoplasias del SNC. De estos casos, entre un $50 \%$ y $70 \%$ fueron gliomas (tumor derivado de células gliales en el SNC), siendo el Glioblastoma Multiforme el subtipo de mayor incidencia, con pronóstico muy reservado por sus características histológicas y grado IV como mayor malignidad. 
Los tumores cerebrales también pueden variar por su origen: los gliomas por ejemplo, se forman a partir del tejido conectivo del cerebro; los meningiomas, se forman a partir de células que constituyen las meninges; y los linfomas, los cuales mayoritariamente inciden en personas inmunosuprimidas. Por su parte los tumores de tipo metastásico, se originan a partir de otros tumores localizados en otras partes del cuerpo y que se trasladan a los tejidos cerebrales. A pesar de sus diversas etiologías, la sintomatología comúnmente descrita es similar según Lezak (2004): cefaleas, náuseas, focos epilépticos, trastornos sensitivos y motores, alteraciones endocrinas y deterioro en funciones cognitivas. Asimismo, con el transcurso del tiempo, se incrementan una serie de cambios neurofisiológicos y anatómicos como el aumento de la presión intracraneana, edema cerebral, el desplazamiento de estructuras cerebrales, destrucción de tejido cerebral y vías de comunicación (fascículos), entre otros, siendo la combinación de estas alteraciones lo que incide directamente en el deterioro del funcionamiento cognitivo y la afectación de la calidad de vida y la funcionalidad de la persona.

Lezak (2004) menciona que, durante el inicio de la patología tumoral, las manifestaciones conductuales podrían ser confundidas como sintomatología psiquiátrica y ser diagnosticadas erróneamente. Se presentan, por ejemplo, alteraciones en el estado de ánimo, mayor nivel de ansiedad, desorganización conductual y del pensamiento, disminución de la iniciativa, apatía, depresión e irritabilidad, entre otras que son, en realidad, cambios neuro-conductuales y de regulación emocional propios de los cambios a nivel neurológico.

Por otro lado, algunas de las manifestaciones cognitivas más comunes son: déficit en procesos de atención y concentración, fallos de memoria de trabajo, disfunción ejecutiva, apraxia, alteraciones visoconstructivas, disminución de la velocidad de procesamiento y alteraciones de lenguaje (Dwan et al., 2015; Schagen et al., 2014; Rojas, Lorenzana, Luviano, Yánez, Ruíz \& Hernández, 2007; Lezak, 2004).
En el escenario de secuelas posibles descrito, desde la psicología, uno de los aportes más valiosos para el trabajo multidisciplinario lo constituye la valoración neuropsicológica. Esta como herramienta que permite conocer, describir y cuantificar la actividad cognoscitiva y el desempeño de las funciones cognitivas de una persona. Sin embargo, como señala SalazarVillanea (2012), no se trata únicamente de contrastar el rendimiento contra baremos internacionales o nacionales de puntuaciones típicas, sino de la adecuación de las tareas y la comprensión del impacto del déficit para evitar experiencias de frustración y construir el andamiaje necesario para el logro de las metas establecidas por las personas en sus contextos de desarrollo familiar, académico, laboral o comunitario.

Señala la autora que debe procederse por observación, formulación y comprobación de hipótesis clínicas durante ejercicios o pruebas concretas y, en sí misma, la valoración neuropsicológica supone una considerable experiencia clínica y un profundo nivel de conocimientos sobre la anatomía y el funcionamiento cerebral. En esta línea, insiste, tiene el potencial para permitir un mejor ajuste entre habilidades personales y demandas del entorno, posibilitando entrenamientos para afrontar la vida diaria con las habilidades preservadas y la mejor recuperación posible de su nivel de rendimiento previo (Salazar-Villanea, 2012).

Alvarán et al. (2008) aportan que la evaluación neuropsicológica cumple con tres objetivos: verificar la existencia de trastornos cognitivos relacionados con el daño cerebral, determinar la magnitud relativa del daño e identificar la habilidad de la persona para regresar y ajustarse a su contexto previo. Para esto, Alvarán et al. (2008) también detallan cuatro aspectos importantes sobre las características de los tumores cerebrales que recomiendan tener presentes al momento de la evaluación neuropsicológica: 1) localización del tumor; 2) tamaño del tumor; 3) invasión del tejido cerebral; 4) velocidad de crecimiento del tumor. Todos estos aspectos inciden no solo en el contexto específico de la administración de la valoración neuropsicológica, sino también en el eventual programa de rehabilitación 
cognitiva y en las estrategias de adaptación y ajuste de la persona a su entorno.

Como afirma Salazar-Villanea (2012), el alto coste de las secuelas y desajustes en las diferentes esferas psicosociales donde la persona con patología cerebral tumoral se desenvuelve, implica también una labor clínica y psicoeducativa que la psicología debe realizar con la familia y comunidad. Igualmente, los y las profesionales en psicología deben considerar la necesidad de implementar planes de rehabilitación cognitiva, ya que en todos los casos, la plasticidad cerebral y la reorganización tras una lesión implica la capacidad del cerebro para adaptarse a la nueva situación.

Junqué y Mataró, desde el 2000, afirmaban que el posicionamiento internacional de la neuropsicología debe comprenderse como el resultado de la integración de profesionales en psicología al trabajo de la neurocirugía. Estos autores enfatizan que ambas disciplinas mantienen, desde su inicio, una relación simbiótica e interdisciplinaria, que se gestó a partir de la observación de las secuelas de las lesiones cerebrales, luego de las intervenciones neuroquirúrgicas. Es a partir de esta relación, y junto con el surgimiento de las neurociencias, que se reconoce un predominio esencial por el factor neurofuncional, más allá de un factor anatómico de las estructuras del SNC, sobre la calidad de vida (Duffau, 2006).

Todas estas líneas de acción profesional que han sido descritas previamente, asociadas al rol de la psicología y la neuropsicología clínica, son las que se han incorporado en una visión dialógica con otras áreas de la salud para dar sustento a la experiencia clínico-formativa que se documenta en este trabajo. Son, en ese sentido, la base teórica que fundamenta la experiencia de colaboración entre la Universidad de Costa Rica y el Hospital México, con la inclusión de estudiantes avanzados de último año de la Licenciatura en la carrera de Psicología bajo la asesoría técnica, la supervisión clínica y la dirección de un equipo multidisciplinario de profesionales con formación especializada en psicología clínica, neuropsicología clínica, psicología de la salud, psicología de la familia, neurocirugía, psiquiatría, radiología y enfermería.

Las metas clínico-formativas de esta experiencia que se sistematiza han incluido: a.) la participación dentro del proceso de atención y diagnóstico de personas portadoras de tumores cerebrales; b.) el acompañamiento en la atención psicológica y emocional a pacientes y familiares; c.) la formación en las sesiones clínicas del equipo multidisciplinario; d.) la colaboración en valoraciones psicológicas y neuropsicológicas en las etapas pre, trans y post quirúrgicas (con administración de protocolos de evaluación neuropsicológica pre y post operatorias, así como una aplicación trans-operatoria para conocer mejor el perfil cognitivo del paciente despierto durante los diferentes momentos del proceso quirúrgico de resección tumoral) y e) la contribución en la atención a familiares, a través de sesiones psicoeducativas, elaboración de materiales y talleres sobre los efectos y secuelas cognitivas probables, su manejo y ajuste a la vida cotidiana (Ortega, 2013; Ortiz, 2014; Quesada, 2015; Molinari, 2015).

Adicionalmente, se ha coordinado la posibilidad de seguimiento y acompañamiento domiciliar de los pacientes y sus familias desde las prácticas profesionalizantes supervisadas en el Módulo de Neuropsicología de la Escuela de Psicología de la Universidad de Costa Rica, a cargo de la Dra. Salazar Villanea. De esta forma, se llevan a cabo, además, algunos procesos post-quirúrgicos de acompañamiento, contención emocional y fortalecimiento de estrategias compensatorias para la adaptación de las capacidades actuales del usuario(a) en su cotidianidad, mejorando su calidad de vida.

Es así como esta iniciativa, ya con cuatro años en desarrollo, ha permitido una exitosa acogida tanto a nivel universitario como hospitalario, generando la necesidad de sostenibilidad y continuidad. Dado que este trabajo representa una iniciativa pionera a nivel centroamericano, es un ejemplo de la capacidad de la psicología para insertarse en espacios no tradicionales en Costa Rica y tiene sus antecedentes en amplias prácticas mundialmente reconocidas y validadas. A 
continuación se describen algunos ejemplos de casos atendidos en esta experiencia clínico-formativa en contexto hospitalario, ilustrando sus posibles alcances y limitaciones.

\section{Método}

Desde el año 2012 han sido atendidos más de treinta casos con valoraciones psicológicas y neuropsicológicas, según el protocolo hospitalario establecido para la toma de decisiones en el equipo multidisciplinario de este proyecto clínico-formativo. Sin embargo, para este trabajo se han seleccionado cuatro casos clínicos que permitan ejemplificar el proceso de atención y evaluación pre y post operatorias, así como el tipo de evidencia derivada.

El protocolo de atención contempló, en todos los casos, entrevistas, observaciones clínicas y subpruebas de valoración psicológica y neuropsicológica que fueron definidas con criterio clínico y científico, basado en: a) una exhaustiva revisión bibliográfica sobre evidencia de su uso a nivel internacional; b) el criterio experto de varios especialistas consultados en acuerdo y consenso y c) la experiencia clínica de los y las profesionales del equipo multidisciplinario que trabajan en el proyecto y según los protocolos establecidos en los manuales de procedimientos de los diversos servicios de atención del Hospital México.

Las subpruebas fueron extraídas de variadas baterías y test neuropsicológicos que se utilizan, tanto internacional como nacionalmente, en los protocolos diagnósticos hospitalarios y que eran ya parte de los instrumentos utilizados en el manual de procedimientos del Servicio de Psiquiatría y Psicología del Hospital México. Fueron elegidos en sesión de equipo clínico considerando para cada caso la función cognitiva de interés, la relación con la patología tumoral cerebral y la historia o evolución específica.

\section{Descripción de los casos}

Los cuatro casos seleccionados forman parte de una población de usuarios hospitalarios con tumores cerebrales que implicaban compromiso frontal o cambios ejecutivos, los cuales ingresaron al Servicio de
Neurocirugía del Hospital México, durante el período comprendido entre agosto de 2012 a junio de 2014. De interés es señalar que estos casos corresponden a personas adultas jóvenes, por lo que las implicaciones para su calidad de vida a largo plazo resultan críticas y hacen pertinente su ejemplificación.

La valoración para su selección implicó un abordaje multidisciplinario. La persona usuaria debía ser evaluada por un equipo profesional compuesto por neurocirugía, anestesia, psiquiatría y psicología. Lo anterior con el fin de garantizar las condiciones idóneas de seguridad e integridad tanto del paciente como del equipo profesional que participaba del procedimiento quirúrgico.

Los casos atendidos que se describen cumplían con los siguientes criterios de inclusión: contaban con un desempeño a nivel cognitivo valorado como "suficiente" para poder responder a las consignas y para ser candidatos a una cirugía como paciente despierto (para recibir estimulación cortical y subcortical cerebral); brindaban su consentimiento informado para la atención o tratamientos y participaban de manera completamente voluntaria. Como criterio de exclusión se consideró la presencia de patología dual o padecimientos psiquiátricos, pues los pacientes no podían tener historia previa de trastornos mentales graves y prolongados, esquizofrenia y otros trastornos psicóticos; delirium o demencia.

A continuación, en la tabla 1, se presentan los datos generales de interés de los cuatro casos de pacientes con diagnóstico de neoplasia, con un rango de edad entre 32 y 43 tres años (media de 36.75 años y 14.25 años de escolaridad en educación formal, que corresponden a una formación superior a la secundaria y algunos estudios técnicos o universitarios).

\section{Instrumentos}

Para los casos seleccionados se documentan estudios de tomografía axial computarizada (TAC) así como de resonancias magnéticas (RM), que fueron realizados a los y las pacientes para poder confirmar el diagnóstico de neoplasia cerebral. 
Tabla 1

Descripción general: variables de interés de los 4 casos

\begin{tabular}{|c|c|c|c|c|c|c|c|}
\hline Sujeto & Edad & $\begin{array}{c}\text { Dominancia } \\
\text { manual } \\
\text { (lateralidad) }\end{array}$ & Tipo de tumor & $\begin{array}{c}\text { Localización } \\
\text { del tumor }\end{array}$ & $\begin{array}{c}\text { Presencia } \\
\text { de secuelas } \\
\text { funcionales } \\
\text { de la lesión }\end{array}$ & $\begin{array}{c}\text { Consumo de } \\
\text { anticonvulsivantes }\end{array}$ & $\begin{array}{c}\text { Manifiesta } \\
\text { conciencia de } \\
\text { enfermedad } \\
\text { asociada a } \\
\text { secuelas }\end{array}$ \\
\hline 1 & 36 & Derecha & Oligodendroglioma & $\begin{array}{c}\text { Parieto- } \\
\text { occipital } \\
\text { izquierdo }\end{array}$ & Sí & No & Sí \\
\hline 2 & 36 & Derecha & Glioblastoma & $\begin{array}{l}\text { Fronto- } \\
\text { parietal } \\
\text { derecho }\end{array}$ & Sí & Sí & Sí \\
\hline 3 & 32 & Izquierda & Glioblastoma & $\begin{array}{l}\text { Fronto- } \\
\text { temporal } \\
\text { izquierdo }\end{array}$ & Sí & Sí & Sí \\
\hline & & & & Frontal & & & \\
\hline 4 & 43 & Derecha & Glioblastoma & Izquierdo & $\mathrm{No}$ & No & Sí \\
\hline
\end{tabular}

Cada uno de los pacientes en los casos descritos, así como sus familiares, fueron entrevistados por el equipo multidisciplinario. Se realizó una entrevista clínica y examen mental, así como la administración de un protocolo de subpruebas neuropsicológicas. Este protocolo está formado por un total de 22 subtests y subpruebas de: Programa Integrado de exploración neuropsicológica o Test de Barcelona (Peña-Casanova, 2005), Escala de Inteligencia de Wechsler para adultos WAIS-IV, Evaluación Neuropsicológica Breve en español Neuropsi, Trail Making Test y Test Stroop de Colores y Palabras (Golden, 2001 ). Se utilizaron los puntajes brutos de estas pruebas y se realizó una evaluación cualitativa de los procesos y funciones cognitivas de atención, memoria, lenguaje, habilidades visoespaciales y funciones ejecutivas.

Fue también necesaria, en dos de los casos que se describen a continuación, la evaluación de sintomatología asociada a depresión y ansiedad con la Escala Hospitalaria de Depresión y Ansiedad (HAD), adaptación colombiana (Rico, Restrepo \&
Molina, 2005) de la versión española de la HAD. Dicha escala está compuesta por 14 ítems, creada por Zigmond y Snaith (1983). Es utilizada para detectar posibles indicadores de trastornos de ansiedad y depresión, especialmente en contextos hospitalarios y psiquiátricos. Su estructura consiste en una escala tipo Likert que va de un rango de 0 a 3 , a través de la cual los pacientes describen los sentimientos y pensamientos que han percibido durante la última semana. Consta de dos subescalas de depresión y ansiedad compuestas por 7 ítems intercalados; la puntuación entre 0-7 se considera normal, de 8-10 dudoso, y de 11 o más, un problema clínico (Rico, et al., 2005).

La Escala EuroQol como medida de autopercepción del estado de salud también fue utilizada en esos dos casos por criterio del equipo, dadas sus condiciones para responder apropiadamente más allá de la verbalización de sus percepciones personales en entrevista. Se hizo uso del sistema descriptivo, así como de la escala visual analógica. El primero está compuesto por cinco preguntas que evalúan cinco 
dimensiones de calidad de vida: movilidad, cuidado personal, actividades cotidianas, dolor/malestar y ansiedad/depresión. Cada una de las preguntas posee tres opciones de respuesta, que oscilan de 1-3; (1) "no tengo problemas", (2) "tengo problemas moderados" y (3) "tengo muchos problemas". El segundo consiste en la evaluación del paciente de su estado de salud en una escala que comprende desde 0 (peor estado de salud posible) a 100 (mejor estado de salud posible) (Ministerio de Sanidad y Política Social de Madrid, 2009).

Los instrumentos anteriormente citados fueron seleccionados para complementar la evaluación con la entrevista clínica y el protocolo neuropsicológico, dado su amplio uso en contextos hospitalarios, su fácil comprensión y rápida aplicación, considerando, así, las limitaciones funcionales de pacientes con neoplasias cerebrales.

\section{Procedimiento}

Los casos que se describen fueron sometidos, según el protocolo del Hospital México, a las tres fases de valoración durante el internamiento hospitalario: una fase pre-operatoria, una trans-operatoria y otra postoperatoria. Durante la fase preoperatoria, la persona es evaluada inicialmente por el personal de neurocirugía, donde se realizan los exámenes neurológicos necesarios para el diagnóstico preciso del tipo de lesión. Posteriormente, el equipo de anestesiología, psiquiatría y psicología realizan sus respectivas valoraciones y entrevistas para determinar si la persona cumple los criterios de inclusión y exclusión clínicos y médicos necesarios para considerarse como candidata para la cirugía en modalidad despierto.

Específicamente el o la profesional en psicología realiza, en una primera sesión, una entrevista clínica al paciente y a sus familiares o cuidadores para comprender de una manera integral la historia de la persona, sus condiciones premórbidas, rendimiento, expectativas, sintomatología y funcionalidad actual. De ser posible, según la urgencia de la cirugía y el estado del paciente, se evalúa por medio de un auto-reporte el nivel de ansiedad/depresión y nivel calidad de vida percibida. Esta sesión se complementa con el respectivo acompañamiento emocional y entrenamiento básico en técnicas de relajación progresiva y respiración, así como la presentación de materiales psicoeducativos que explican los procedimientos a los cuales serán sometidos, con estímulos visuales y auditivos como herramientas de anticipación para potenciar los recursos de manejo y afrontamiento del procedimiento quirúrgico. Una segunda sesión consiste en la valoración neuropsicológica a través del protocolo anteriormente descrito, con el objetivo de perfilar el nivel de rendimiento de los principales procesos cognitivos y establecer una línea base de funcionamiento con aquellas habilidades alteradas y preservadas.

La fase trans-operatoria se refiere al momento de la cirugía donde la persona se mantiene despierta a lo largo del procedimiento neuroquirúrgico de resección tumoral, en este se efectúa la estimulación cortical, acompañada de la evaluación neuropsicológica. Durante este proceso, se brinda el respectivo apoyo psicológico y acompañamiento emocional a la persona (en este manuscrito no se incluyen los detalles sobre esta fase en particular).

Esta modalidad de cirugía despierto permite al profesional en neurocirugía, con el apoyo del profesional en neuropsicología, determinar las llamadas áreas elocuentes que hacen referencia a las funciones del control motor cortical y procesos de lenguaje que deben preservarse primordialmente.

Finalmente, la fase postoperatoria consiste en un periodo aproximadamente de tres a seis meses posteriores a la cirugía, en la que se valora nuevamente a la persona y evalúan su rendimiento neuropsicológico, auto-reporte sobre las posibles secuelas, sintomatología de estado de ánimo, ansiedad o depresión y el nivel de calidad de vida percibida subjetivamente. La variabilidad del tiempo transcurrido para esta valoración se asocia a factores diversos de recuperación médica, posibilidad de traslados en citas de seguimiento o ambos. Esta fase permite hacer un contraste de la valoración inicial con lo manifestado posteriormente por la persona y por sus familiares, determinando, así, los posibles cambios a nivel cognitivo y su impacto tanto a nivel emocional 
como en su calidad de vida. La información recabada sienta el inicio del apoyo del proceso de rehabilitación cognitiva y el posible uso de estrategias compensatorias para el manejo de las secuelas.

\section{Resultados}

Se presentan los datos generales del rendimiento neuropsicológico documentado de los cuatro casos seleccionados, según los puntajes obtenidos en la valoración previa a la cirugía (evaluación pre-operatoria) y posterior a la misma (evaluación post-operatoria). Asimismo, se describen los datos obtenidos por medio de las escalas HAD y EuroQol, en aquellos casos que se incluyó su aplicación. La selección de las subpruebas y escalas que se administraron correspondió a una decisión clínica según el estado y sintomatología de los y las pacientes, la urgencia de intervención quirúrgica, sus condiciones de funcionamiento y el criterio consensuado del equipo multidisciplinario según el protocolo establecido hospitalariamente. Para la presentación de los datos, se tomaron en cuenta los puntajes brutos obtenidos, la cantidad de aciertos y errores, así como las respuestas perseverativas e intrusiones presentadas durante la evaluación. También se incluyen las notas clínicas cualitativas que corresponden a las anotaciones realizadas en el expediente por parte del equipo multidisciplinario.

A continuación se presentan los datos caso por caso, junto con una breve caracterización.

Sujeto 1: mujer de 36 años de edad, diestra, laboraba como dependiente en un negocio y se encontraba desempleada. Acude al Servicio de Neurocirugía del Hospital México con historia de un mes de evolución de deterioro cognitivo y funcional, caracterizado por cefaleas de predominio occipital, alteraciones en su memoria, trastorno de la marcha y tremor distal en extremidades de predominio derecho. Se documentan crisis convulsivas recurrentes, trastornos visuales y refiere visión borrosa. La figura 1 muestra los resultados de la Resonancia Magnética y la tabla 2 presenta el resumen de sus puntuaciones.

La paciente reporta verbalmente en la entrevista de su valoración post-operatoria una percepción personal y subjetiva de mayor calidad de vida, con mejor funcionalidad autónoma y con disminución de la sintomatología física.

Sujeto 2: femenino, 36 años de edad, diestra, educadora de profesión. Acude al servicio de neurocirugía por lesión espacio ocupante frontoparietal derecha, dificultades motoras y deterioro en el rendimiento cognitivo. La figura 2 muestra el resultado de la Resonancia Magnética preoperatoria y la tabla 3 presenta el resumen de sus puntuaciones.

La paciente reporta en su valoración postoperatoria una percepción subjetiva general de mejora en su propio rendimiento cognitivo. Manifiesta que su rendimiento mejora con el uso de estrategias compensatorias que le han permitido beneficiarse para procesos complejos como resolución de problemas, regulación de su conducta y llevar acabo secuencias en sus labores rutinarias.

Sujeto 3: masculino de 32 años, dominancia manual izquierda, se desempeña como cajero bancario. Es remitido del servicio de emergencias del Hospital México al servicio de neurocirugía, debido a síncope con pérdida de la conciencia como consecuencia de un episodio de crisis convulsiva. La figura 3 permite observar los resultados de la resonancia magnética de cráneo pre-operatoria, que muestra una lesión espacio ocupante fronto-temporal izquierda; ver figura $3 \mathrm{y}$ tabla 4 con el resumen de sus puntuaciones.

El paciente reporta en su valoración postoperatoria una percepción subjetiva de mejor rendimiento con más velocidad en la resolución de tareas diarias y laborales; así como menor sintomatología ansiosa y depresiva.

Sujeto 4: masculino, 43 años, dominancia manual derecha, asistente judicial. Acude al servicio de neurocirugía por lesión espacio ocupante frontal izquierda. Al momento de la evaluación, reporta que es capaz de realizar todas sus actividades básicas e instrumentales de la vida diaria. Preserva adecuadamente su funcionalidad social y la toma de decisiones, pero percibe dificultades atencionales en actividades académicas, así como olvidos ocasionales durante sus funciones laborales. 
Tabla 2

Sujeto 1: rendimientos en evaluación pre-operatoria y post-operatoria

\begin{tabular}{|c|c|c|c|}
\hline Subpruebas & $\begin{array}{l}\text { Noviembre } \\
2013\end{array}$ & $\begin{array}{c}\text { Mayo } \\
2014\end{array}$ & $\begin{array}{l}\text { Notas clínicas cualitativas del evaluador } \\
\text { (evaluación } 6 \text { meses después) }\end{array}$ \\
\hline \multicolumn{4}{|l|}{ Atención } \\
\hline Atención - Dígitos Directos & 4 & 4 & $\begin{array}{l}\text { Se mantiene el desempeño en tareas de dígitos } \\
\text { directos. }\end{array}$ \\
\hline Secuencias Automáticas (Directos) & 5 & 5 & $\begin{array}{l}\text { Se conserva el rendimiento en secuencias } \\
\text { automáticas. }\end{array}$ \\
\hline TMT (A) & $300 \mathrm{~s}$ & $218 s$ & $\begin{array}{l}\text { Mejora el rendimiento al disminuir el tiempo de } \\
\text { ejecución de la prueba, sin embargo se observan } \\
\text { aún alteraciones en el control atencional. }\end{array}$ \\
\hline \multicolumn{4}{|l|}{ Memoria } \\
\hline Secuencias Automáticas (Indirectos) & 1 & 2 & \\
\hline Codificación Curva Aprendizaje & 4 & 6 & $\begin{array}{l}\text { Muestra una leve mejoría en la curva de aprendizaje } \\
\text { de palabras. }\end{array}$ \\
\hline Recuerdo Memoria Verbal Espontánea & 0 & 2 & Mejora levemente en las pruebas de recuerdo \\
\hline Reconocimiento Memoria Verbal & 10 & 12 & espontáneo y reconocimiento. \\
\hline Memoria Trabajo Dígitos Inversos & 3 & 2 & Se mantiene dificultades en dígitos inversos. \\
\hline Figura de Rey Memoria & 2 & 3 & $\begin{array}{l}\text { Se mantiene el rendimiento en la reconstrucción } \\
\text { visoespacial. }\end{array}$ \\
\hline \multicolumn{4}{|l|}{ Lenguaje } \\
\hline Repetición Verbal Oraciones & 59 & 60 & \multirow{5}{*}{$\begin{array}{l}\text { Se observa un rendimiento general que se mantiene } \\
\text { en comprensión, producción y denominación, } \\
\text { con leve aumento en el desempeño en la fluidez } \\
\text { semántica y fonológica. }\end{array}$} \\
\hline Comprensión Verbal Ordenes & 16 & 16 & \\
\hline $\begin{array}{l}\text { Comprensión Verbal } \\
\text { Palabras (Imágenes) }\end{array}$ & 6 & 6 & \\
\hline Fluidez Verbal Semántica & 6 & 8 & \\
\hline Fluidez Verbal Fonológica & 2 & 6 & \\
\hline Comprensión Lectora Ordenes & 5 & $\mathrm{~N} / \mathrm{A}$ & $\begin{array}{l}\text { Reporta visión borrosa, por lo que no se pudo } \\
\text { aplicar la prueba. }\end{array}$ \\
\hline \multicolumn{4}{|l|}{ Habilidades Visoespaciales } \\
\hline Figura de Rey Copia & 7 & 8 & Se mantienen sus rendimientos. \\
\hline \multicolumn{4}{|l|}{ Funciones Ejecutivas } \\
\hline TMT (B) & $780 \mathrm{~s}$ & $442 \mathrm{~s}$ & $\begin{array}{l}\text { Se observan indicios de mejoría en el rendimiento } \\
\text { asociado a inhibición de interferencias. }\end{array}$ \\
\hline Stroop $(C)$ & 14 & 62 & \multirow{3}{*}{$\begin{array}{l}\text { Mantiene preservada su habilidad para inhibir la } \\
\text { interferencia, mejorando su rendimiento. }\end{array}$} \\
\hline Stroop $(\mathrm{P})$ & 29 & 55 & \\
\hline Stroop (PC) & 16 & 23 & \\
\hline
\end{tabular}




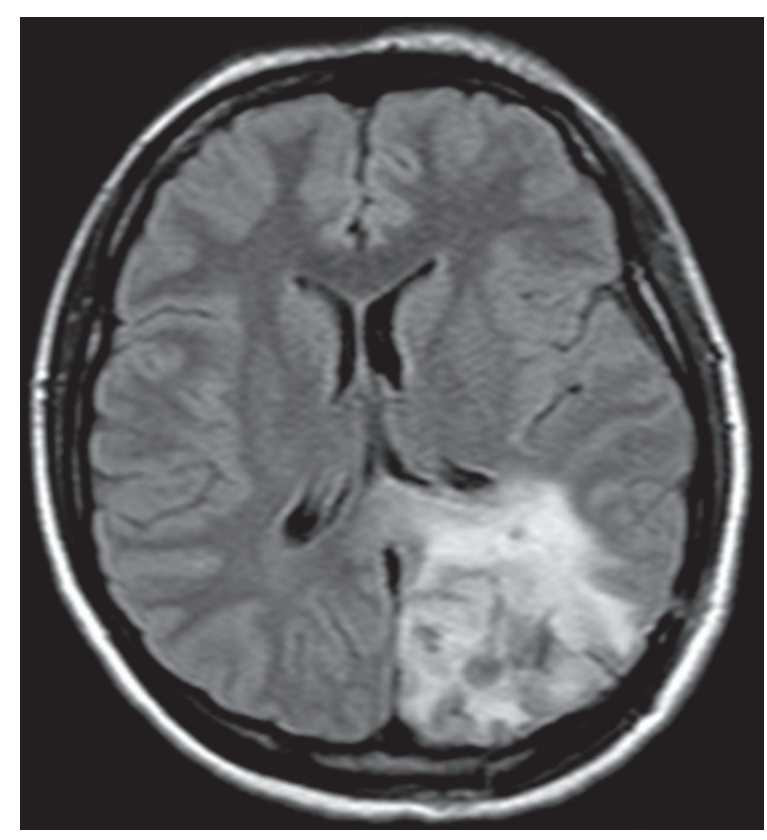

Figura 1. Sujeto 1. Resonancia magnética secuencia T2 pre-operatoria con lesión parieto-occipital izquierda con compromiso del cuerpo calloso.

La figura 5 permite observar los resultados del TAC post-operatorio de la lesión frontal- izquierda, en el tabla 5 y figura 6 se presentan el resumen de sus puntuaciones.

El paciente reporta, en su valoración post-operatoria, una percepción subjetiva general de estabilidad y leve mejoría en sus rendimientos atencionales y dominio cognitivo, en el cual reportaba la mayor afectación antes del procedimiento neuroquirúrgico. Manifiesta, sin embargo, cambios en su regulación emocional con mayores dificultades para la inhibición y el manejo de la sintomatología ansiosa y depresiva cuando se enfrenta a tareas complejas que le producen frustración.

\section{Discusión}

En la diversidad de los perfiles de rendimiento, deterioro físico y neuropsicológico reportados en los casos descritos, debe considerarse la influencia de factores asociados como: variabilidad interindividual, localización y extensión de la lesión, etiología del tumor y tiempo de evolución, nivel premórbido de funcionamiento, reserva cognitiva y motivacional, simetría anatómica y funcional, la lateralidad e incluso el nivel de consciencia del déficit y el uso de estrategias compensatorias en la vida diaria según el apoyo familiar.

Sin embargo, aún con la diversidad de posibles variables intervinientes, en esta experiencia clínicoformativa de atención hospitalaria es relevante la evidencia documentada de afectaciones en el funcionamiento cognitivo de los pacientes costarricenses, principalmente asociadas a alteraciones en procesos atencionales, memoria de trabajo, curvas de aprendizaje, velocidad de procesamiento de la información, fluidez verbal y funcionamiento ejecutivo. Ello es consistente con lo reportado internacionalmente donde, tras la resección cerebral tumoral con cirugías despiertos, los rendimientos parecen tender a estabilizarse y mejorar leve y temporalmente después de la intervención neuroquirúrgica; aunque las secuelas permanezcan con la evolución de la enfermedad (Schagen et al., 2014; RojasRomán, et al. 2007; Duffau, 2006). En esa línea, Dwan, et al. (2015) insisten en la importancia del seguimiento, 
Tabla 3

Sujeto 2. Rendimientos en evaluación pre-operatoria y post-operatoria

\begin{tabular}{|c|c|c|c|}
\hline Subpruebas & $\begin{array}{l}\text { Noviembre } \\
2012\end{array}$ & $\begin{array}{l}\text { Diciembre } \\
2013\end{array}$ & $\begin{array}{l}\text { Notas clínicas cualitativas del evaluador } \\
\text { (evaluación } 13 \text { meses después) }\end{array}$ \\
\hline \multicolumn{4}{|l|}{ Atención } \\
\hline Atención - Dígitos Directos & 7 & 5 & $\begin{array}{l}\text { Disminuye la puntuación directa en tareas de dígitos } \\
\text { directos. }\end{array}$ \\
\hline Secuencias Automáticas (Directos) & 5 & 5 & Se conserva el rendimiento en secuencias automáticas. \\
\hline TMT (A) & $50 \mathrm{~s}$ & $49 \mathrm{~s}$ & $\begin{array}{l}\text { No se presentan cambios evidentes en el tiempo de } \\
\text { ejecución de la prueba, se observan aún alteraciones } \\
\text { en el control atencional. }\end{array}$ \\
\hline \multicolumn{4}{|l|}{ Memoria } \\
\hline Secuencias Automáticas (Indirectos) & 5 & 4 & \multirow[t]{2}{*}{ No se observan diferencias evidentes. } \\
\hline Codificación Curva Aprendizaje & 10 & 10 & \\
\hline $\begin{array}{l}\text { Recuerdo Memoria } \\
\text { Verbal Espontánea }\end{array}$ & 12 & 12 & Se mantiene el rendimiento. \\
\hline Reconocimiento Memoria Verbal & 12 & 12 & \\
\hline Memoria Trabajo Dígitos Inversos & 4 & 5 & Mejora en una cifra los dígitos inversos. \\
\hline \multicolumn{4}{|l|}{ Lenguaje } \\
\hline $\begin{array}{l}\text { Lenguaje Espontáneo } \\
\text { Descripción de Lámina }\end{array}$ & 6 & 6 & Mantiene sus rendimientos. \\
\hline Repetición Verbal Oraciones & 60 & 60 & \multirow{6}{*}{$\begin{array}{l}\text { Se conserva un buen rendimiento en pruebas } \\
\text { de comprensión, repetición, y denominación, } \\
\text { mostrando una mejoría en semejanzas y pruebas de } \\
\text { fluidez de categorías semánticas y fonológicas. }\end{array}$} \\
\hline Comprensión Verbal Órdenes & 16 & 16 & \\
\hline $\begin{array}{l}\text { Comprensión Verbal } \\
\text { Palabras (Imágenes) }\end{array}$ & 12 & 12 & \\
\hline Abstracción Semejanzas & 30 & 32 & \\
\hline Fluidez Verbal Semántica & 23 & 34 & \\
\hline Fluidez Verbal Fonológica & 0 & 6 & \\
\hline \multicolumn{4}{|l|}{ Funciones Ejecutivas } \\
\hline TMT (B) & $139 \mathrm{~s}$ & $69 \mathrm{~s}$ & $\begin{array}{l}\text { Mejora evidente en la reducción del tiempo de } \\
\text { ejecución con mayor velocidad de procesamiento. }\end{array}$ \\
\hline Stroop $(\mathrm{C})$ & 96 & 100 & \multirow{3}{*}{$\begin{array}{l}\text { Se observan indicios de mejoría en el rendimiento } \\
\text { asociado a inhibición de interferencias. }\end{array}$} \\
\hline Stroop $(\mathrm{P})$ & 73 & 71 & \\
\hline Stroop (PC) & 34 & 40 & \\
\hline
\end{tabular}

para que las personas con tumores cerebrales puedan ser sometidas a varios ciclos de evaluaciones en diferentes momentos y puedan compararse los rendimientos conforme progresa su condición y se hacen necesarios nuevos tratamientos o intervenciones neuroquirúrgicas.
Si bien es claro que un mayor deterioro cognitivo y funcional podría afectar a estas personas con la progresión de su enfermedad, el reconocimiento de los déficits tempranos y la intervención neuropsicológica para la rehabilitación de sus funciones cognitivas 
60 Salazar, Ortega, Ortiæ, Esquivel, Vindas \& Montero

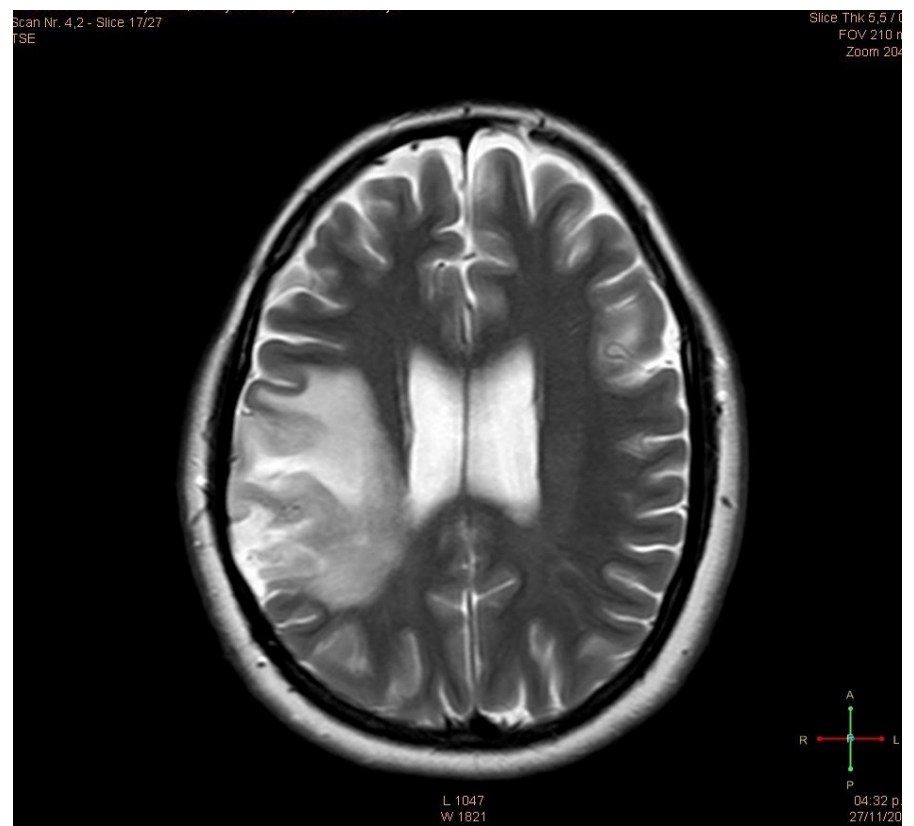

Figura 2. Sujeto 2. Resonancia magnética pre-operatoria con lesión fronto- parietal derecha.

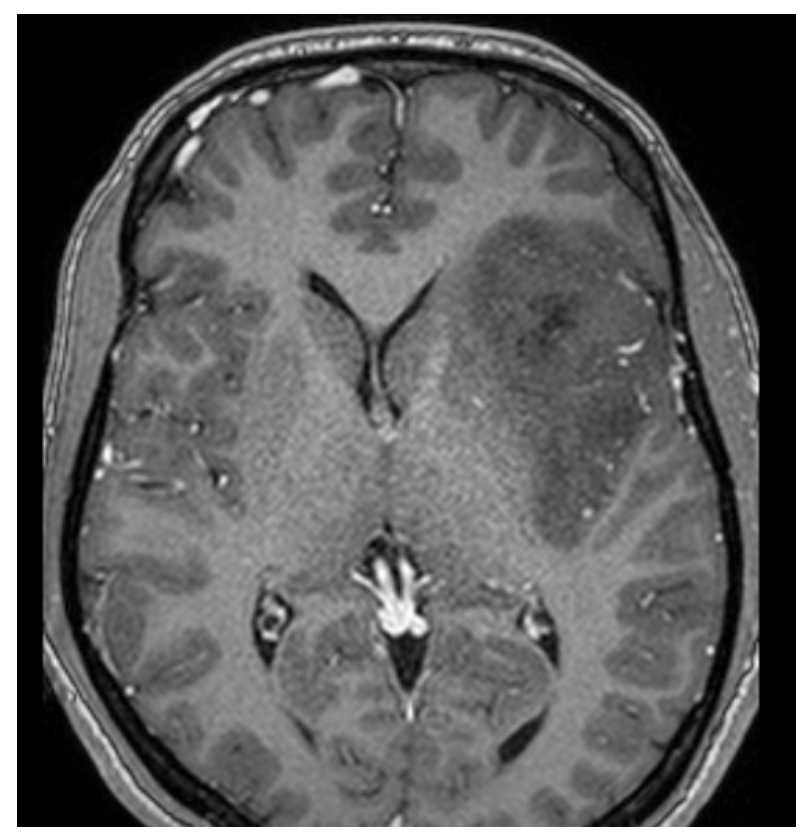

Figura 3. Sujeto 3. Resonancia de cráneo pre-operatoria con lesión fronto-temporal izquierda. 
Tabla 4

Sujeto 3. Rendimientos en evaluación pre-operatoria y post-operatorio

\begin{tabular}{|c|c|c|c|}
\hline Prueba & $\begin{array}{l}\text { Abril } \\
2014\end{array}$ & $\begin{array}{l}\text { Julio } \\
2014\end{array}$ & Notas clínicas cualitativas del evaluador (evaluación 3 meses después) \\
\hline \multicolumn{4}{|l|}{ Atención } \\
\hline Atención - Dígitos Directos & 3 & 5 & Indicios de mejoría en sus puntuaciones en tareas de dígitos directos. \\
\hline Secuencias Automáticas (Directos) & 6 & 5 & No se aprecian diferencias evidentes en el rendimiento. \\
\hline TMT (A) & $27 \mathrm{~s}$ & $26 s$ & $\begin{array}{l}\text { No se presentan cambios importantes en el tiempo de ejecución de la } \\
\text { prueba. }\end{array}$ \\
\hline \multicolumn{4}{|l|}{ Memoria } \\
\hline Secuencias Automáticas (Indirectos) & 5 & 6 & $\begin{array}{l}\text { No se aprecian diferencias evidentes en el rendimiento a pesar de la } \\
\text { leve mejora. }\end{array}$ \\
\hline Codificación Curva Aprendizaje & 8 & 6 & Disminuye la cantidad de palabras. \\
\hline Recuerdo Memoria Verbal Espontánea & 6 & 6 & Se conserva el rendimiento previo. \\
\hline Reconocimiento Memoria Verbal & 12 & 12 & \\
\hline Memoria Trabajo Dígitos Inversos & 3 & 4 & Mejora en una cifra los dígitos inversos. \\
\hline Figura Rey Memoria & 7,5 & 10 & Mejora la cantidad de detalles que recuerda. \\
\hline \multicolumn{4}{|l|}{ Lenguaje } \\
\hline Repetición de Errores Semánticos & 8 & 8 & Mantiene el rendimiento. \\
\hline $\begin{array}{l}\text { Lenguaje Espontaneo } \\
\text { Descripción de Lámina }\end{array}$ & 6 & 6 & \\
\hline Repetición Verbal Oraciones & 60 & 59 & \\
\hline Comprensión Verbal Ordenes & 14 & 16 & Indicios de leve mejoría. \\
\hline Comprensión Lectora Ordenes & 12 & 12 & Se conserva el mismo rendimiento mostrado en las pruebas anteriores. \\
\hline $\begin{array}{l}\text { Comprensión Verbal } \\
\text { Palabras (Imágenes) }\end{array}$ & 12 & 12 & \\
\hline $\begin{array}{l}\text { Escritura Espontanea } \\
\text { Denominación Escrita }\end{array}$ & 6 & 6 & \\
\hline Denominación Verbo-Verbal & 6 & 6 & \\
\hline Abstracción Semejanzas & 29 & 28 & No se presentan cambios. \\
\hline Fluidez Verbal Semántica & 25 & 22 & Disminuye la cifra de palabras recordadas. \\
\hline Fluidez Verbal Fonológica & 8 & 9 & No difiere de forma evidente el puntaje. \\
\hline Reconocimiento de Objetos & 6 & 6 & Se conserva el mismo puntaje. \\
\hline Repetición Verbal Logatomos & 6 & 8 & Indicios de leve mejoría. \\
\hline \multicolumn{4}{|l|}{ Funciones Ejecutivas } \\
\hline TMT (B) & $112 \mathrm{~s}$ & $59 \mathrm{~s}$ & $\begin{array}{l}\text { Indicios de mejoría en la reducción del tiempo de ejecución con mayor } \\
\text { velocidad de procesamiento. }\end{array}$ \\
\hline Stroop $(\mathrm{C})$ & 100 & 135 & \multirow{3}{*}{$\begin{array}{l}\text { Se observan indicadores de mejoría en el rendimiento las tres pruebas } \\
\text { del Stroop. }\end{array}$} \\
\hline Stroop $(\mathrm{P})$ & 67 & 91 & \\
\hline Stroop (PC) & 56 & 61 & \\
\hline \multicolumn{4}{|l|}{ Habilidades Viso-espaciales } \\
\hline Figura Rey Copia & 9,5 & 10,5 & No se presentan cambios relevantes. \\
\hline
\end{tabular}




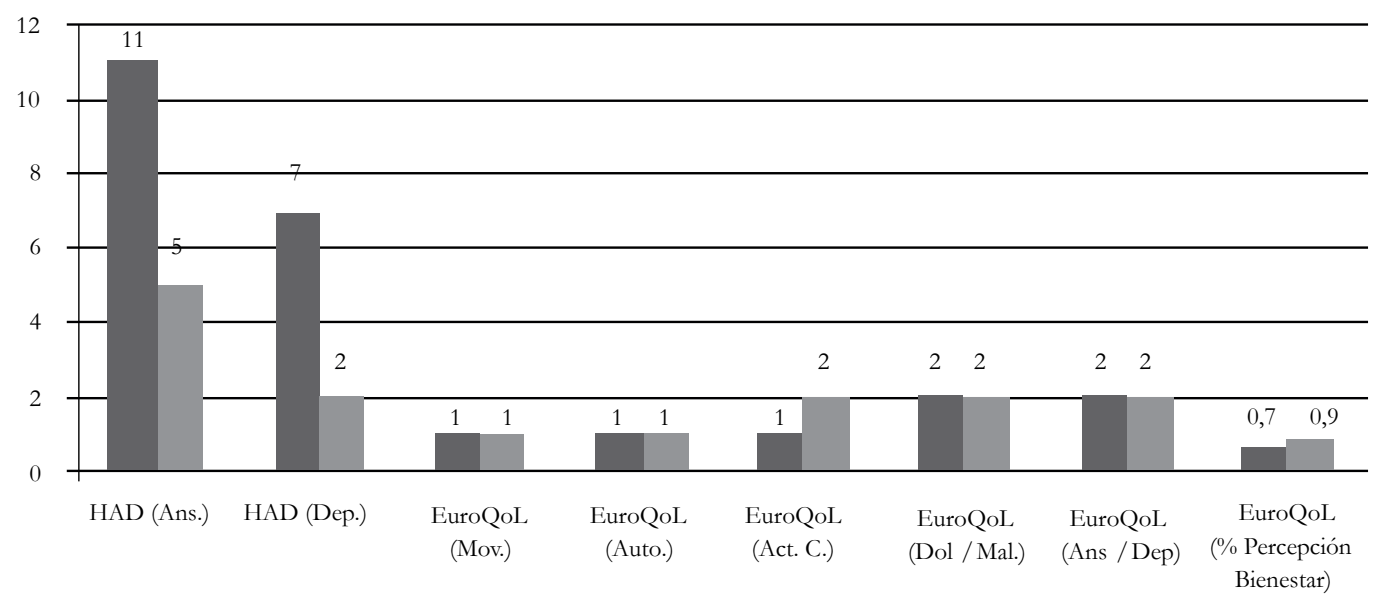

Figura 4. Sujeto 3. Resultados en escalas HAD y EuroQol pre-operatoria y post-operatoria.

podrían incidir directa y positivamente en su calidad de vida (Lezak, 2004; Schagen et al., 2014). Identificar afectaciones en este funcionamiento brinda elementos adicionales al proceso de toma de decisión a nivel médico y familiar, permitiendo mayor claridad sobre el tipo de cuidado requerido, los retos para quienes brindarán dichos cuidados en el entorno familiar y el ajuste a las demandas de la cotidianidad de las personas según su nivel de secuelas.

Por otro lado, en la misma línea de los hallazgos de Rojas et al. (2007), los auto-reportes de los y las pacientes permiten comprender que los signos de ansiedad y depresión encontrados en algunos casos parecen asociarse con la capacidad de reconocer sus déficits. Sin embargo, tiende a disminuir con el uso de estrategias y con la percepción de estabilidad o leve mejoría en sus rendimientos, funcionalidad y calidad de vida tras la intervención neuroquirúrgica.

En este sentido, el valor de la intervención psicológica y neuropsicológica en cuanto al acompañamiento, psicoeducación y adquisición de estrategias de compensación de los déficits, resulta fundamental y trasciende la labor de valoración en la que se logra documentar esa leve, pero consistente, tendencia a la mejoría en los rendimientos cognitivos posterior a la resección tumoral. Ello en coherencia con las expectativas de los y las pacientes y sus familiares, quienes valoran la posibilidad de prolongación de la vida con calidad y opciones de desarrollo personal y social.

En el proceso de colaboración multidisciplinario y clínico-formativo de la Escuela de Psicología de la Universidad de Costa Rica y el Hospital México desde el 2012, se ha logrado evidenciar la relevancia de describir el perfil cognitivo de rendimiento en los diferentes momentos del proceso de atención neuroquirúrgico. Lo anterior valorando los cambios y condiciones de las funciones cognitivas afectadas y preservadas $y$ acompañando cercanamente al paciente y su familia; de manera que incida positivamente sobre la calidad de vida de las personas. De esta forma, se construye, también, la base para la posible estructuración de un programa futuro de rehabilitación neuropsicológica, enfocado en recomendaciones y estrategias ajustadas de forma individualizada para la inserción de las personas a los diferentes contextos de desarrollo de su vida (familiar, académico, laboral y comunitario).

Son, entonces, al menos tres los niveles de impacto que la sistematización de la experiencia en Costa Rica y los casos atendidos han permitido observar hasta ahora: a.) impacto en el macro nivel científico-académico, para el posicionamiento de la neuropsicología clínica como especialidad fundamental y la generación de una base de evidencia que apoye la toma de decisiones neuroquirúrgicas; b.) impacto en el meso nivel de 


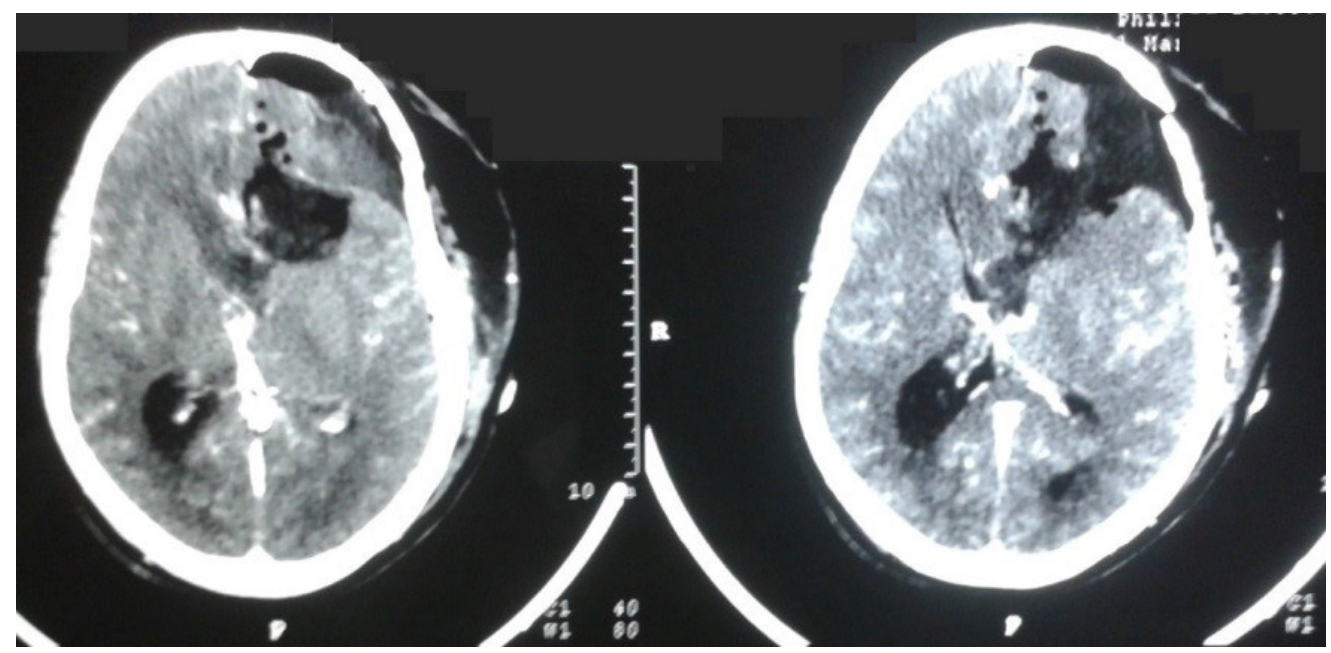

Figura 5.Sujeto 4. Tomografía de cráneo post-operatoria de la resección tumoral de una lesión frontal izquierda.

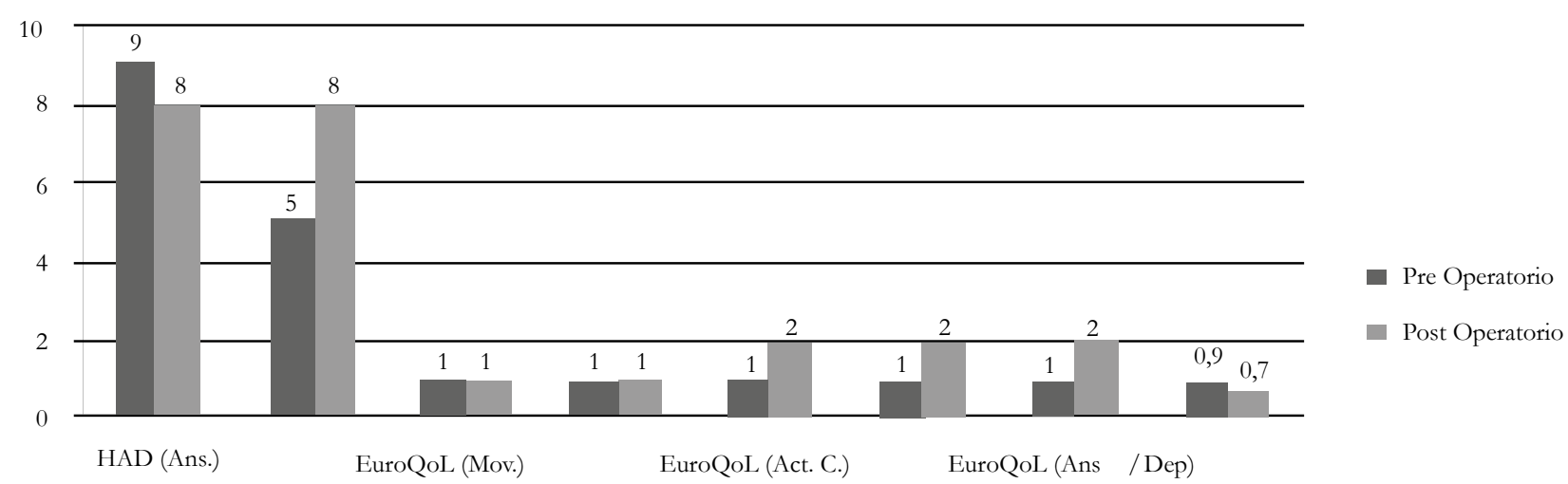

Figura 6. Sujeto 4. Resultados en escalas HAD y EuroQol pre-operatoria y post-operatoria.

los alcances de las relaciones multidisciplinarias en contextos hospitalarios, para brindar un mejor servicio de atención y c.) impacto en el micro nivel del beneficio para la calidad vida de los y las pacientes y sus familias. Todo ello, fundamentado en antecedentes clínicos e investigaciones internacionales que cuentan ya con mayor evidencia empírica.

En esa línea, como parte de las limitaciones que deben reconocerse, figura la necesidad de promover en Costa Rica procesos de investigación clínica longitudinal y análisis cuantitativos de los datos con las mediciones repetidas que esta experiencia en marcha vaya documentando, para ampliar esa base de evidencia empírica que a nivel internacional es extensa y que, en Costa Rica, es aún incipiente. La ausencia de instrumentos de valoración psicológica y neuropsicológica estandarizados para Costa Rica, con baremos adaptados, limita además la comparación y manejo de los datos. Por otro lado, existe, a nivel 
Tabla 5

Sujeto 4: rendimientos en evaluación pre-operatoria y post-operatoria

Prueba Marzo Julio Notas clínicas cualitativas del evaluador (evaluación 4 meses después)

$2014 \quad 2014$

\begin{tabular}{|c|c|c|c|}
\hline \multicolumn{4}{|l|}{ Atención } \\
\hline Atención - Directos & 3 & 3 & Conserva el desempeño en tareas de dígitos directos. \\
\hline Secuencias Automáticas (Directos) & 5 & 6 & No se aprecian diferencias en el rendimiento. \\
\hline TMT (A) & $42 \mathrm{~s}$ & $52 \mathrm{~s}$ & $\begin{array}{l}\text { Presenta un leve incremento en el tiempo que requiere para realizar la } \\
\text { tarea. }\end{array}$ \\
\hline \multicolumn{4}{|l|}{ Memoria } \\
\hline Secuencias Automáticas (Indirectos) & 4 & 5 & No se aprecian diferencias relevantes. \\
\hline Codificación Curva Aprendizaje & 4 & 2 & Disminuye la cantidad de palabras. \\
\hline Evocación Memoria V. Espontanea & 4 & 3 & Disminuye la cantidad de palabras que recuerda de manera espontánea. \\
\hline Reconocimiento Memoria Verbal & 7 & 7 & Se conserva la capacidad de recordar las palabras a través de claves. \\
\hline Memoria Trabajo Dígitos Inversos & 2 & 3 & Mejora en una puntuación directa los dígitos inversos. \\
\hline Figura Rey Memoria & 5 & 11,5 & Mejora significativa en la cantidad de detalles que recuerda. \\
\hline \multicolumn{4}{|l|}{ Lenguaje } \\
\hline Repetición de Errores Semánticos & 8 & 8 & Conserva el mismo rendimiento. \\
\hline $\begin{array}{l}\text { Lenguaje Espontáneo } \\
\text { (Descripción de Lámina) }\end{array}$ & 5 & 6 & \\
\hline Repetición Verbal Oraciones & 57 & 58 & El rendimiento no presenta cambios evidentes. \\
\hline Comprensión Verbal Ordenes & 16 & 14 & Disminuye con respecto a la primera vez. \\
\hline Comprensión Lectora Ordenes & 12 & 10 & Disminuye la cantidad de órdenes que atiende. \\
\hline $\begin{array}{l}\text { Comprensión Verbal } \\
\text { Palabras (Imágenes) }\end{array}$ & 12 & 12 & $\begin{array}{l}\text { Se conserva el mismo rendimiento previo en comprensión, escritura y } \\
\text { denominación. }\end{array}$ \\
\hline $\begin{array}{l}\text { Escritura Espontanea } \\
\text { Denominación Escrita }\end{array}$ & 6 & 6 & \\
\hline Denominación Verbo-Verbal & 6 & 6 & \\
\hline Abstracción Semejanzas & 15 & 21 & Es capaz de nombrar mayor cantidad de semejanzas. \\
\hline Fluidez Verbal Semántica & 13 & 12 & No hay diferencias evidentes. \\
\hline Fluidez Verbal Fonológica & 0 & 1 & \\
\hline Reconocimiento de Objetos & 6 & 6 & \\
\hline Repetición Verbal Logatomos & 7 & 8 & Su rendimiento se mantiene. \\
\hline \multicolumn{4}{|l|}{ Funciones Ejecutivas } \\
\hline TMT (B) & $443 \mathrm{~s}$ & $162 \mathrm{~s}$ & $\begin{array}{l}\text { Indicios de mejoría evidente en la reducción del tiempo de ejecución con } \\
\text { mayor velocidad de procesamiento. }\end{array}$ \\
\hline Stroop $(\mathrm{C})$ & 87 & 107 & Se observan indicadores de mejoría en el rendimiento del Stroop. \\
\hline Stroop $(\mathrm{P})$ & 60 & 78 & \\
\hline Stroop (PC) & 36 & 24 & Disminución de la supervisión e inhibición para realizar la tarea. \\
\hline \multicolumn{4}{|l|}{ Habilidades Viso-espaciales } \\
\hline Figura Rey Copia & 10 & 11.5 & No se presentan cambios evidentes. \\
\hline
\end{tabular}


nacional una carencia, de profesionales en psicología con entrenamiento en neuropsicología y rehabilitación cognitiva dentro del sistema de salud, razón por la cual la presente experiencia es una alianza interinstitucional que permite subsanar de forma limitada la atención a las personas afectadas por la patología cerebral tumoral y sus secuelas sobre la calidad de vida.

Por último, es relevante rescatar que este proyecto ha buscado garantizar un impacto psicosocial con intervenciones psicoeducativas y preparación cognitiva y emocional para los proceso quirúrgicos. Incluso, ha logrado la extensión de acciones sociales-comunitarias para la atención con rehabilitación cognitiva y entrenamiento en uso de estrategias compensatorias a través de la vinculación de espacios de docencia-clínica e investigación.

Un reto claro que se deriva de la incipiente evidencia empírica positiva asociada a este modelo de atención clínica multidisciplinaria, es la demanda de una atención similar en otras patologías neurológicas como lo son los traumas craneoencefálicos y los accidentes cerebrales vasculares entre otros. En ese sentido, hay una clara necesidad de sostener y desarrollar iniciativas más amplias a nivel nacional. También constituye un reto la posibilidad de registrar y analizar empíricamente, con mayor rigurosidad científica, los datos clínicos que en los expedientes hospitalarios se van documentando en la práctica clínico-formativa, de tal manera que la divulgación de resultados permita mejores decisiones en el ámbito de la salud y mayor beneficio para los y las pacientes, así como para sus familiares.

\section{Reconocimientos}

Se agradece la colaboración del servicio de neurocirugía del Hospital México, especialmente a la Dra. Dessiré Marie Gutiérrez Gutiérrez y a la M.Sc. Ericka Torres Porras por su apoyo al proyecto.

\section{Referencias}

Alvarán, L., Gómez, L., Aguirre, D., \& Ortiz, L. (2008). Caracterización neuropsicológica de pacientes con glioma tratados en el Instituto de Cancerología de Medellín. Medellín: Universidad de Antoquia.
Brannon, L. (2007). Health psychology. (7th ed.). Belmont: Wadsworth.

Duffau, H. (2006). New concepts in surgery of WHO grade II gliomas: functional brain mapping, connectionism and plasticity- a review. Journal of Neuro-Oncology 79(1), 77-115.

Dwan, T. M., Ownsworth, T., Chambers, S., Walker, D. G., \& Shum, D. H. (2015). Neuropsychological assessment of individuals with brain tumor: comparison of approaches used in the classification of impairment. Frontiers in Oncology, 5:56. doi: 10.3389/fonc. 2015.00056

Esquivel, M., Vargas, E., Alonso, J., Fernández, J., Jiménez, R., \& Cabezas, C. (2012). Características epidemiológicas de la población adulta con diagnóstico de gliomas atendida en los Hospitales Metropolitanos de San José, Costa Rica durante los años 2010-2012. Neuroeje 25(1), 39-46.

Golden, C. J. (2001). Stroop-Test de Colores y Palabras. Madrid: TEA Ediciones.

Junqué, C., \& Mataró, M. (2000). La simbiosis neurocirugía-psicología en el origen de la neuropsicología. Neurocirugía 11(2), 82-88. doi: 10.1016/S1130-1473(00)70741-X

Lezak, M. (2004). Neuropsychological Assessment. (4 ed.). New York: Oxford University Press.

Ministerio de Sanidad y Política Social (2009). Estudios sobre la calidad de vida de pacientes afectados por determinadas patologias. Recuperado de: http://www. msssi.gob.es/organizacion/sns/planCalidadSNS/ docs/Estudios_calidad_vida_pacientes.pdf

Molinari, M. (2015). Evaluación neuropsicológica en pacientes con tumores cerebrales del Servicio de Neurocirugia del Hospital México. Trabajo Final de graduación para optar por el grado de Licenciatura en Psicología. Universidad de Costa Rica.

Organización Mundial de la Salud. (2012). Globocan: Estimated Cancer Incidence, Mortality and Prevalence Worldwide in 2012. Recuperado de: http://globocan. iarc.fr/Pages/fact_sheets_cancer.aspx 
Ortega-Araya, L.E. (2013). La inserción y rol del psicólogo en el ambiente hospitalario: aproximación a las necesidades de la práctica clínica psicológica en el Servicio de Neurocirugía y la Unidad de Neuro-Oncología del Hospital México. Trabajo Final de Graduación para optar por el grado de Licenciatura en Psicología. Universidad de Costa Rica.

Ortiz, J. (2014). Inserción profesional de la Psicología en el ambiente hospitalario y la práctica clínica en el Servicio de Neurocirugia y la Unidad de Neuro-Oncología del Hospital México. Trabajo Final de Graduación para optar por el grado de Licenciatura en Psicología. Universidad de Costa Rica.

Peña Casanova, C. (2005). Programa integrado de exploración neuropsicológica Test de Barcelona. Barcelona, España. Masson.

Quesada, M. J. (2015). Implementación de un programa de acompañamiento psicológico y psicoeducación para pacientes con patología tumoral cerebral y sus familiares en el Servicio de neurocirugia del Hospital México. Trabajo Final de Graduación para optar por el grado de Licenciatura En Psicología. Universidad de Costa Rica.

Rico, J. L., Restrepo, M., \& Molina, M. (2005). Adaptación y Validación de la Escala Hospitalaria de Ansiedad y Depresión (HAD) en una muestra de pacientes con cáncer del Instituto Nacional de Cancerología de Colombia. Avances en Medición 3, 73-86.

Rojas-Román, S. I., Lorenzana-Galicia, R. D., Luviano, L., Yánez, G., Ruíz-García, E., \& Hernández-
Gutiérrez, L. (2007). Evaluación neuropsicológica pre y posquirúrgica de pacientes con tumor cerebral frontal. Archivo de Neurociencias, 1(12), 14-24.

Salazar-Villanea, M. (2012). Neuropsicología y envejecimiento: el potencial de la memoria autobiográfica en investigación e intervención clínica. Revista Costarricense de Psicología 31(1-2), 123-146.

Schagen, S. B., Klein, M., Reijneveld, J. C., Brain, E., Deprez, S., Joly, F., Scherwath, A., Schrauwem, W., \& Wefel, J. S. (2014). Monitoring and optimising cognitive function in cancer patients: present knowledge and future directions. European Journal of Cancer Care, 12(1),29-40.

Shedden-Mora, M. (2014). Introducción a la práctica basada en evidencias en el área de la Psicología Clínica y Salud. Material didáctico del curso impartido en la Universidad de Costa Rica. Auspiciado por el Departamento de Psicología Clínica y Psicoterapia Escuela de Psicología, Universidad de Hamburgo, Alemania.

Spring, B. (2007). Evidence-based practice in clinical psychology: What it is, why it matters; what you need to know. Journal of Clinical Psychology, 63(7), 611-631.

Zigmond, A. S. \& Snaith, R. P. (1983). The Hospital Anxiety and Depression Scale. Acta Psychiatric Scandinavic 67(6), 361-370. 